\title{
A Cystic Clival Chordoma with CT and MRI Unconventional Appearances
}

\author{
Pamela Guadalupi ${ }^{1,2}$ Marco Gessi ${ }^{3} \quad$ Luca Massimi $i^{4,5}$
}

Massimo Caldarelli ${ }^{4,5}$ Simona Gaudino ${ }^{1}$

\author{
Address for correspondence Pamela Guadalupi, MD, UOC \\ Radiodiagnostica e Neuroradiologia, Dipartimento di Diagnostica per \\ Immagini, Radioterapia, Oncologia ed Ematologia, Fondazione \\ Policlinico Universitario A. Gemelli IRCCS, 00168, Rome, Italy \\ (e-mail: pamela.guadalupi@gmail.com).
}

\begin{abstract}
Keywords

- chordoma

- CT

- cystic

- extradural

- MRI

- transdural

We present the first case of clival cystic chordoma with extradural location, transdural transgression, and moderate bone involvement in a 10 -year-old girl. Chordoma showed unconventional appearances on computed tomography (CT) and magnetic resonance imaging (MRI), due to cystic components, extradural space location with extensive intradural extension, moderate superficial bone involvement. Surgery confirmed the extradural location and histopathological examination revealed cystic chordoma. MRI and $\mathrm{CT}$ findings were not characteristic for a single lesion; differential diagnoses included cystic lesions such as epidermoid and dermoid cyst, ecchordosis physaliphora, and benign notochordal cell tumors.
\end{abstract}

\section{Introduction}

A clival cystic chordoma is an extremely rare entity and represents a radiological challenge. If this already rare entity deviates from the classic computed tomography (CT) and magnetic resonance imaging (MRI) appearances of chordomas due to the disproportion between the large extraosseous soft tissue component and the moderate bone involvement, and also it deviates from the few reported cases of cystic intracranial chordoma due to the extradural location, an inappropriate differential pathway may be led down. We present a case of clival chordoma with $\mathrm{CT}$ and MRI unconventional appearances, such as cystic aspect, extradural location, and poor bone involvement.

\section{Case Report}

A 10-year-old girl was admitted to our institution after the radiological diagnosis of a mass of the clival region. The clinical history started several months before the admission with headache, pain on the right side of the head mainly raised by physical activity (especially sport). Because of this persisting symptom and because of the appearance of right palpebral spasms, the young girl underwent an published online

February 28, 2022
DOI https://doi.org/ 10.1055/s-0041-1741044. ISSN 0971-3026.

\footnotetext{
(C) 2022. Indian Radiological Association. All rights reserved. This is an open access article published by Thieme under the terms of the Creative Commons Attribution-NonDerivative-NonCommercial-License, permitting copying and reproduction so long as the original work is given appropriate credit. Contents may not be used for commercial purposes, or adapted, remixed, transformed or built upon. (https://creativecommons.org/ licenses/by-nc-nd/4.0/) Thieme Medical and Scientific Publishers Pvt. Ltd., A-12, 2nd Floor, Sector 2, Noida-201301 UP, India
} 

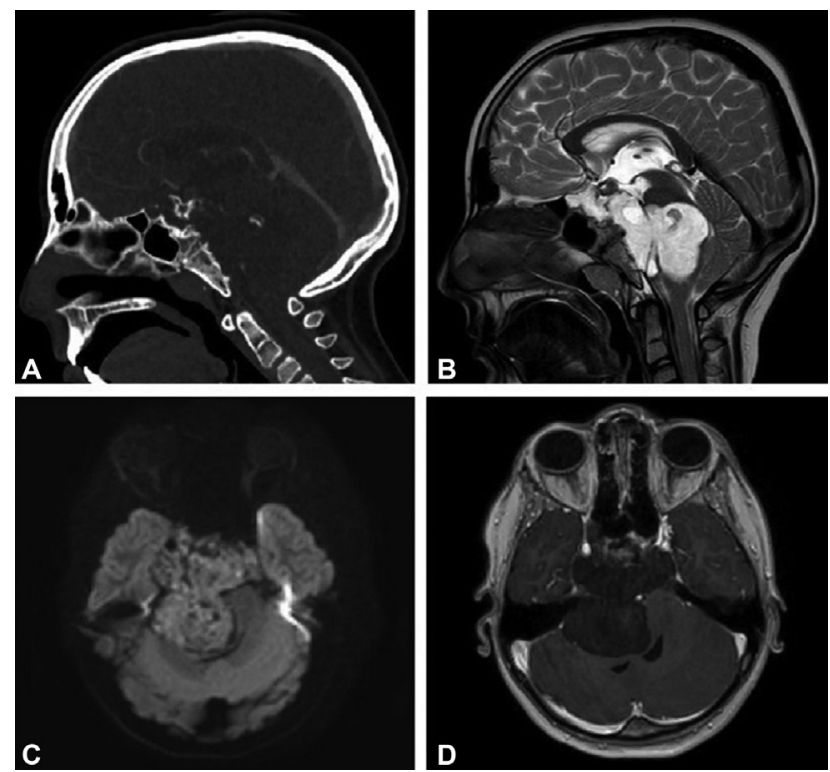

Fig. 1 (A-D) Sagittal reformatted CT (A) and sagittal T2-WI (B) an expansile retroclival mass. On T2-WI, mass appeared in the extradura space, with transdural passage and intradural component indenting the pons. On CT (A), bone invasion was relatively light, sella turcica, and pituitary gland were elevated by the mass, and a tooth-shaped calcification was floating in the posterior part of the chordoma. Chordoma had a cystic appearance, hyperintense on T2-WI (B), and moderate hyperintense on DWI (C). On T1-WI after gadolinium (D), there was no evidence of contrast enhancement. CT, computed tomography; DWI, diffusion-weighted imaging; T1-WI, T1-weighted imaging; T2-WI, T2-weighted imaging.

ophthalmological evaluation with diagnosis of right eye strabismus (esophoria). After the surgical correction of the strabismus, the headache improved but not disappeared. A right neurosensorial hypoacusia, present since the infancy, showed a worsening instead, hence an MRI was performed. The physical examination at the admission did not point out deficits except for a mild weakness of the left lower limb. The girl was attending normal school and showed normal cognitive and psychomotor development on specific test administered at the admission. Noncontrast-enhanced CT scan showed a centrally located retroclival large mass, extending to the sellar region, with a tooth-shaped calcification floating in the posterior part and a sequestration of destroyed bone in the upper part, resembling the sella, and elevating the pituitary gland. Despite the large dimension of the mass, only a slight bone erosion of the clivus was present (- Fig. 1). MRI better delineated location and extent of the lesion, along the dorsal aspect of the clivus in the extradural space, extending from the foramen magnum to the sellar region. Sagittal T2-weighted imaging (T2-WI) disclosed transdural transgression and invasion of prepontine and right cerebellopontine (CP) angle cistern, with compression and "indention" of the pons, and elevation of the pituitary gland. Axial images showed invasion of both cavernous sinus and Meckel's caves and lateral displacement of cavernous internal carotid arteries. The mass showed low signal intensity on T1-weighted imaging (T1-WI) and very hyperintense signal on T2-WI (-Fig. 1), without evidence of intralesional septa.
Two intralesional foci of increased signal on T1-WI and decreased signal on T2-WI and susceptibility-weighted images (SWI) corresponded to calcifications on CT. Diffusion-weighted images (DWI) demonstrated moderate high signal intensity of the lesion, due to "T2 shine through" (apparent diffusion coefficient [ADC]: $1,400 \times 10^{-6} \mathrm{~mm}^{2} / \mathrm{s}$ in average). After administration of gadolinium-based contrast medium, the mass did not demonstrate any contrast enhancement. A two-step surgical removal of the tumor mass was planned. As first step, the young girl underwent a gross debulking of the tumor through a retrosigmoid approach (craniotomy) to the right $\mathrm{CP}$ angle. The operation was performed under general anesthesia and with electrophysiological intraoperative monitoring. At surgery, the tumor appeared as a gelatinous mass, plastically growing into the $\mathrm{CP}$ angle cistern and extending into the adjacent cisterns. A marked encasement of the vessels and the cranial nerves by the tumor was evident. However, the dissection of the tumor from the neurovascular structures was facilitated by its soft aspect and by the absence of a significant peritumoral inflammatory reaction. The neoplasm was removed by a microsurgical technique and by suction. The neoplasm was followed and removed up to the junction between the posterior and the middle cranial fossae, where a detachment and perforation of the skull base dura mater was evident as well as the tumor passing from the middle to the posterior fossa. The postoperative course was uneventful. The mild motor deficit of the left lower limb quickly disappeared. The patient was readmitted 3 months after the first operation to allow her to finish the school time. No symptoms occurred in this period, and she was neurologically intact at the admission. MRI showed stable residual mass. The girl underwent a second surgery for the removal of the component of the tumor involving the anterior skull base though an endoscopic endonasal transsphenoidal approach. As expected, the tumor was extradural and occupied the retroclival space. The macroscopic appearance was the same as the previous operation. The postoperative course was uneventful. The patient is now receiving proton radiotherapy on the residual tumor. On histopathological examination, the neurosurgical specimens were composed of mucinous material containing epithelial appearing tumor cells, with monomorphic relatively small round nuclei ( $\mathbf{- F i g . ~ 2 A , ~ B ) ; ~ t h e ~ c e l l s ~ w e r e ~ f o c a l l y ~}$ arranged in cords and clusters. Focally, a small fragment of mature bone tissue within the mucinous stroma was observed. The cells showed expression of vimentin, pan-cytokeratin, and S100 ( - Fig. 2C-E, respectively). The INI1 nuclear expression was retained (not shown). The cells did not display significant mitotic and/or proliferative activity (MIB1 immunostaining) (not shown).

\section{Discussion}

Chordomas are rare and locally invasive malignant tumors that represent $1 \%$ of intracranial tumors and $4 \%$ of all primary bone tumors; they most commonly affect adults in their fourth and fifth decades of life, with male and whites predilection, while they are rare among children. ${ }^{1}$ Arising 

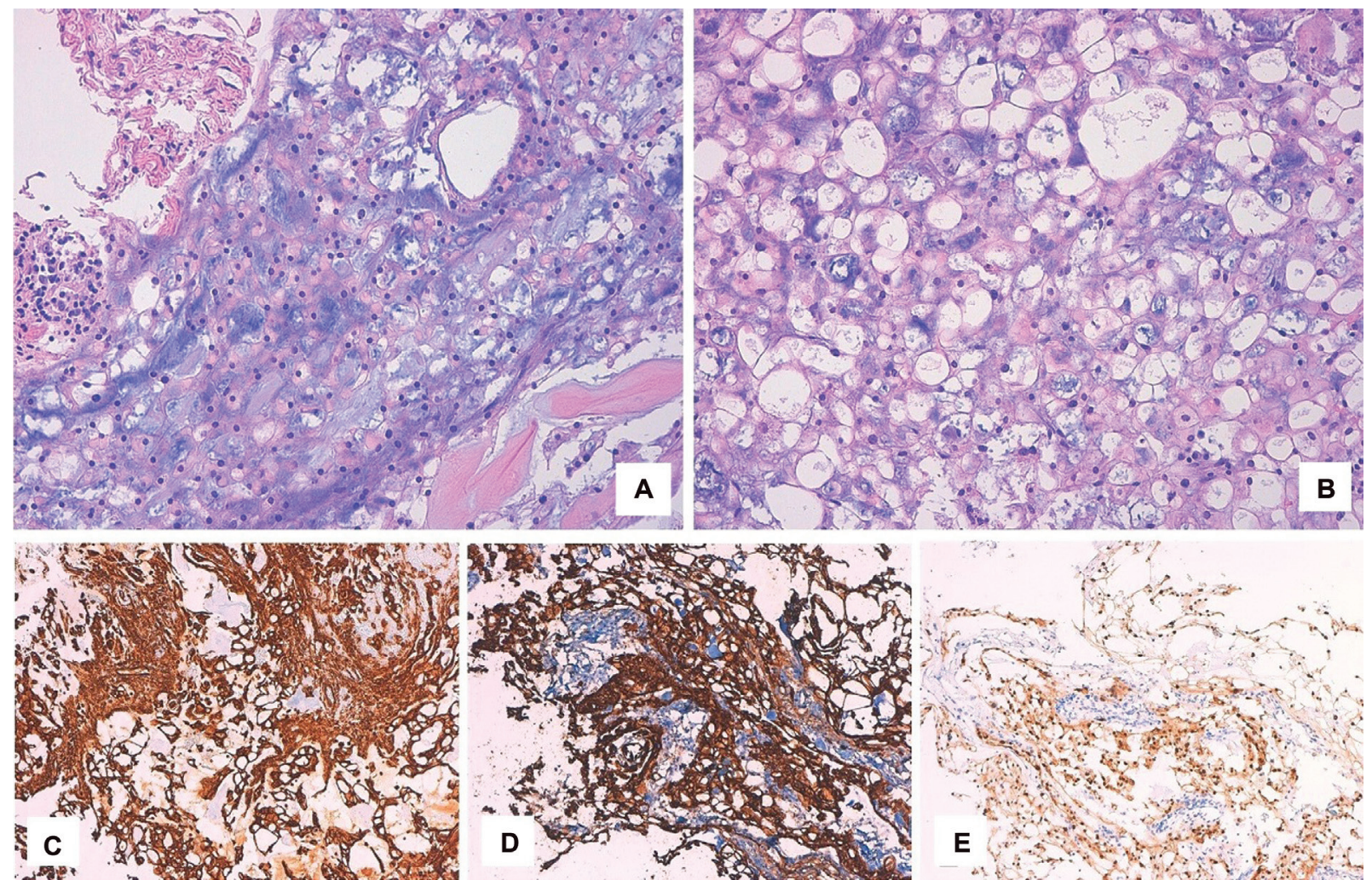

Fig. 2 (A-E) Histopathological features of cystic chordoma. The histopathological examination revealed mucinous material containing monomorphic epithelial tumor cells with small-rounded nuclei and large clear cytoplasm, focally arranged in cords and clusters (A, B). The cells showed expression of vimentin (C), pan-CK (D), and S100 (E). (A, B) Routine $\mathrm{H}$ and E staining; (C): immunostaining with monoclonal mouse antihuman vimentin, clone V9, Dako-Agilent, Cernusco sul Naviglio, Italy; (D): immunostaining with monoclonal mouse antihuman CK antibody, clone AE1/AE3, Dako-Agilent; (E): immunostaining with polyclonal rabbit antibody antihuman S100, Dako-Agilent; all immunohistochemistry performed on Dako-Omnis staining system, Dako. CK, cytokeratin.

from embryonic remnants of the primitive notochord, chordomas can develop along the cranial (32\%), spinal (32.8\%), and sacral $(29.2 \%)$ portions of the craniovertebral axis. ${ }^{1}$ Spheno-occipital synchondrosis is the most common site of origin of intracranial chordomas, arising from the upper third or along the caudal margin of the clivus. ${ }^{1}$ Occasionally, the extrusion of the notochord during the ossification can occur in aberrant locations, and notochord rests may persist into the extradural or intradural spaces. This would allow the chordomas to grow without bony involvement. ${ }^{2}$ There are three histological variants of chordoma: classical, chondroid chordomas, containing hyaline cartilage, ${ }^{1}$ and dedifferentiated chordoma. Dedifferentiated chordoma comprise $<5 \%$ of all chordoma subtypes, and they are a particularly aggressive tumor with a predilection for the pediatric population with a much poorer prognosis. ${ }^{3}$ Chondroid variant often shows a less aggressive clinical behavior than classical chordoma. ${ }^{4}$

Also, the degree of bone invasion and the integrity of dural are independent risk factors for the clinical prognosis of skull base chordoma patients. ${ }^{4}$ Recently, Wu et al $^{5}$ classified clivus chordoma as endophytic (Type I) or exophytic (Type II) based on the degree of skull base bone invasion, and exploring the mechanisms of such bone invasion, they confirmed that the expression level of PTEN (phosphatase and tensin homolog) gene is associated with the degree of bone invasion, and the regulatory molecule transforming growth factor beta 1 may play an important role in bone invasion (Experimental Study on Differences in Clivus Chordoma Bone Invasion: An iTRAQBased Quantitative Proteomic Analysis). The classic appearance of intracranial chordoma at MRI is a well-circumscribed, expansile soft tissue mass, with high signal intensity on T2-WI, intermediate to low signal intensity on T1-WI, moderate to marked contrast enhancement. Variable intralesional dark areas on SWI (susceptibility weighted imaging) or GRE (gradient echo sequences) images are present due to intratumoral hemorrhages or calcifications; however, calcifications (bone sequestra or dystrophic calcifications) are better depicted by CT scan. ${ }^{6}$

This clival chordoma did not conform to the typical CT and MRI appearances of intracranial chordoma, first due to the completely cystic appearance of the mass and the poor bone involvement in the face of a voluminous extrabone component.

Based on cystic appearance, differential diagnosis included epidermoid cyst (EC), ecchordosis physaliphora (EP), and benign notochordal cell tumors (BNCTs), but considering also the location of the lesion, none of these lesions corresponded to our case (-Table 1). ${ }^{7}$ Although some MRI features were compatible with EC, such as T1 and T2 signal intensity and no contrast enhancement, ECs usually show restricted diffusion on DWI (bright hyperintensity), and they are usually intradural lesions $(75 \%),{ }^{8}$ which are not consistent with our case. EP is a congenital, often asymptomatic, benign lesion derived 
Table 1 Imaging characteristics of our case and differential diagnosis

\begin{tabular}{|c|c|c|c|c|c|c|}
\hline & T1-WI & T2-WI & DWI/ADC & $\begin{array}{l}\text { Contrast } \\
\text { enhancement }\end{array}$ & Bone erosion & Location \\
\hline Our case & Low & High & $\begin{array}{l}\text { No restriction } \\
\left(\text { ADC: } 1,400 \times 10^{-6} \mathrm{~mm}^{2} / \mathrm{s}\right)\end{array}$ & No & Mildly & $\begin{array}{l}\text { Extradural, } \\
\text { secondary } \\
\text { intradural }\end{array}$ \\
\hline $\mathrm{EC}$ & Low & High & Restriction & No & No & Intradural \\
\hline EP & Low & High & No restriction & No & No & Intradural \\
\hline BNCTs & Low & High & No restriction & No & No & Intraosseous \\
\hline $\begin{array}{l}\text { Chondrosarcoma } \\
\text { of the skull base }\end{array}$ & $\begin{array}{l}\text { Low/ } \\
\text { intermediate }\end{array}$ & High & $\begin{array}{l}\text { No restriction } \\
(>\text { mean } A D C \text { value: } 2,051 \pm \\
\left.261 \times 10^{-6} \mathrm{~mm}^{2} / \mathrm{s}\right)^{1}\end{array}$ & Yes & $\begin{array}{l}\text { Yes } \\
\text { "ring-and-arc" } \\
\text { calcification }\end{array}$ & $\begin{array}{l}\text { Extradural off } \\
\text { midline }\end{array}$ \\
\hline
\end{tabular}

Abbreviations: ADC, apparent diffusion coefficient; BNCTs, benign notochordal cell tumors; DWI, diffusion-weighted imaging; EC, epidermoid cyst; EP, ecchordosis physaliphora; T1-WI, T1-weighted imaging; T2-WI, T2-weighted imaging.

from intradural notochord remnants, usually located in the midline, such as in the dorsum of the clivus. ${ }^{9}$ However, EP usually arises from the intradural space, on the contrary, our case seems to be an extradural mass with secondary intradural invasion, a growth behavior that belongs to chordomas. ${ }^{10}$ Whether intradural chordomas arise from EP is still under debate; in literature, rare cases of symptomatic EP are described and malignant transformation to chordoma is also contemplated, ${ }^{11}$ but EP remains relatively small in size (2$3 \mathrm{~cm}$ ) and only few cases of EP $>3 \mathrm{~cm}$ have been reported in the literature, considering that "giant EP" is defined $>6 \mathrm{~cm}^{3}$ in size. ${ }^{9,11}$ The substantially larger dimensions do not support the hypothesis that it could be a degenerated EP.

BNCTs are benign intraosseous lesions of notochordal cell origin reported to be found in $20 \%$ of autopsy series in the midline of clivus and vertebral bodies, with increasing frequency of their identification on MRI studies. ${ }^{12}$ BNCTs appear as well-defined intraosseous lesion, and as for EC and $\mathrm{EP}$, signal intensity is low on T1-WI and high on T2-WI, without contrast enhancement, diffusion restriction, or soft tissue extension, but the location is extradural. ${ }^{12}$ We considered the hypothesis of cystic intracranial chordomas, but based on a review of the cases reported in the literature, while the lack of severe bone involvement resulted as a typical feature, the extradural location was never reported. Most of the intracranial cystic chordomas were intradural, in one case, the lesion was located in the right cavernous sinus and in another case, the authors were not completely sure of the location, which nevertheless seemed to be extradural (-Table 2). ${ }^{13-23}$ Also, the patients' age was not conclusive, only $4 / 11$ patients were child or teenagers. Chondrosarcoma could be another possible differential diagnosis for midline chordomas with dural invasion in the presence of intratumoral calcification, but the absence of contrast enhancement did not support completely this hypothesis. ${ }^{24}$ Chondrosarcoma arises in the petroclival fissure, and therefore, it is usually located off midline. ${ }^{24}$ CT may show calcification of the tumor, often in characteristic "rings and arcs"

Table 2 Case reports of cystic chordomas reported in literature

\begin{tabular}{|l|l|l|l|l|l|l|l|}
\hline Authors (year) & Age/sex & T1-WI & T2-WI & DWI & CE & $\begin{array}{l}\text { Bone } \\
\text { erosion }\end{array}$ & Location \\
\hline Niida et al $(1994)^{13}$ & $5 / \mathrm{M}$ & Hypo & Hyper & - & No (CT) & No & Retroc. Intrad. \\
\hline Nishigaya et al $(1998)^{14}$ & $56 / \mathrm{M}$ & Hypo & Hyper & - & Mild & No & Retroc. Intrad. \\
\hline Seung and Kim $(2004)^{15}$ & $12 / \mathrm{F}$ & Hypo & Hyper & Hyper & No & Mild & Retroc. Intrad. \\
\hline Ciarpaglini et al $(2009)^{16}$ & $60 / \mathrm{M}$ & Hypo & Hyper & - & No & No & Retroc. Intrad. \\
\hline Cho et al $(2012)^{17}$ & $32 / \mathrm{M}$ & Hypo & Hyper & Slightly hyper & Small foci & No & Retroc. Intrad. \\
\hline Hashim et al $(2014)^{18}$ & $15 / \mathrm{F}$ & $\begin{array}{l}\text { Hypo } \\
\text { (+foci of hemo.) }\end{array}$ & Hyper & - & No & No & Retroc. Intrad. \\
\hline AlOtaibi et al $(2014)^{19}$ & $51 / \mathrm{M}$ & Hypo & Hyper & - & Mild & No & Retroc. Intrad. \\
\hline Ozek et al $(2018)^{20}$ & $35 / \mathrm{F}$ & Hypo & Hyper & Hyper & No & No & Retroc. Intrad. \\
\hline Vinke et al $(2016)^{21}$ & $11 / \mathrm{M}$ & Hypo & Hyper & - & Mild & No & Retroc. Intrad. \\
\hline Kim et al, $2018^{22}$ & $27 / \mathrm{M}$ & Hypo & Hyper & Hyper & No & No & Retroc. Intrad. \\
\hline Sathe et al $(2019)^{23}$ & 26/M & Hypo & Hyper & No RD & No & Mild & Cavernous sinus \\
\hline
\end{tabular}

Abbreviations: CE, contrast enhancement; CT, computed tomography; hemo., hemosiderin; Hyper, hyperintense; Hypo, hypointense; Intrad., intradural; RD, restricted diffusion; Retroc., retroclival; T1-WI, T1-weighted imaging; T2-WI, T2-weighted imaging. 
(chondroid matrix mineralization). ${ }^{24}$ Furthermore, recent studies demonstrate that DWI may be helpful in the differential diagnosis between chordoma and chondrosarcoma, with chondrosarcoma having a higher average ADC than chordoma. ${ }^{7}$

\section{Conclusion}

Cystic chordoma is an extremely rare entity that should be considered in the differential diagnosis of cystic lesions of the clival region. But in our case, cystic chordoma not only demonstrated unconventional appearance on CT and MRI with respect to intracranial chordoma, and yet it did not conform to reported radiological appearance of intracranial cystic chordoma.

\section{Declaration of Patient Consent}

The authors certify that they have obtained all appropriate patient consent forms. In the form, the patient(s) has/have given his/her/their consent for his/her/their images and other clinical information to be reported in the journal. The patients understand that their names and initials will not be published and due efforts will be made to conceal their identity, but anonymity cannot be guaranteed.

\section{Financial Support and Sponsorship}

Nil.

\section{Conflict of Interest}

None declared.

\section{References}

1 Erdem E, Angtuaco EC, Van Hemert R, Park JS, Al-Mefty O. Comprehensive review of intracranial chordoma. Radiographics 2003;23(04):995-1009

2 Doucet V, Peretti-Viton P, Figarella-Branger D, Manera L, Salamon G. MRI of intracranial chordomas. Extent of tumour and contrast enhancement: criteria for differential diagnosis. Neuroradiology 1997;39(08):571-576

3 Batista KMP, Reyes KYA, Lopez FP, et al. Immunophenotypic features of dedifferentiated skull base chordoma: an insight into the intratumoural heterogeneity. Contemp Oncol (Pozn) 2017;21(04):267-273

$4 \mathrm{Wu}$ Z, Zhang J, Zhang L, et al. Prognostic factors for long-term outcome of patients with surgical resection of skull base chordomas-106 cases review in one institution. Neurosurg Rev 2010;33 (04):451-456

5 Wu Z, Wang L, Guo Z, et al. Experimental study on differences in clivus chordoma bone invasion: an iTRAQ-based quantitative proteomic analysis. PLoS One 2015;10(03):e0119523

6 Santegoeds RGC, Temel Y, Beckervordersandforth JC, Van Overbeeke JJ, Hoeberigs CM. State-of-the-art imaging in human chordoma of the skull base. Curr Radiol Rep 2018;6(05):16
7 Müller U, Kubik-Huch RA, Ares C, et al. Is there a role for conventional MRI and MR diffusion-weighted imaging for distinction of skull base chordoma and chondrosarcoma? Acta Radiol 2016;57(02):225-232

8 Osborn AG, Preece MT. Intracranial cysts: radiologic-pathologic correlation and imaging approach. Radiology 2006;239(03): 650-664

9 Mehnert F, Beschorner R, Küker W, Hahn U, Nägele T. Retroclival ecchordosis physaliphora: MR imaging and review of the literature. AJNR Am J Neuroradiol 2004;25(10):1851-1855

10 Oikawa S, Kyoshima K, Goto T, et al. Histological study on local invasiveness of clival chordoma. Case report of autopsy. Acta Neurochir (Wien) 2001;143(10):1065-1069

11 Lagman C, Varshneya K, Sarmiento JM, Turtz AR, Chitale RV. Proposed diagnostic criteria, classification schema, and review of literature of notochord-derived ecchordosis physaliphora. Cureus 2016;8(03):e547

12 Nishiguchi T, Mochizuki K, Ohsawa M, et al. Differentiating benign notochordal cell tumors from chordomas: radiographic features on MRI, CT, and tomography. AJR Am J Roentgenol 2011;196(03): 644-650

13 Niida H, Tanaka R, Tamura T, Takeuchi S. Clival chordoma in early childhood without bone involvement. Childs Nerv Syst 1994;10 (08):533-535

14 Nishigaya K, Kaneko M, Ohashi Y, Nukui H. Intradural retroclival chordoma without bone involvement: no tumor regrowth 5 years after operation. Case report. J Neurosurg 1998;88(04):764-768

15 Seung WB, Kim SM. Clival cystic chordoma in children with confused magnetic resonance imaging. J Korean Neurosurg Soc 2004;36:422-424

16 Ciarpaglini R, Pasquini E, Mazzatenta D, Ambrosini-Spaltro A, Sciarretta V, Frank G. Intradural clival chordoma and ecchordosis physaliphora: a challenging differential diagnosis: case report. Neurosurgery 2009;64(02):E387-E388, discussion E388

$17 \mathrm{Cho} \mathrm{H,} \mathrm{Yu} \mathrm{IK,} \mathrm{Kim} \mathrm{SM,} \mathrm{Kim} \mathrm{HK.} \mathrm{Magnetic} \mathrm{resonance} \mathrm{imaging} \mathrm{of} \mathrm{the}$ intradural prepontine chordoma mimicking an epidermoid cyst: pathologic correlation. J Korean Soc Radiol 2012;67(02):73-77

18 Hashim H, Rosman AK, Abdul Aziz A, Roqiah AK, Bakar NS. Atypical clival chordoma in an adolescent without imaging evidence of bone involvement. Malays J Med Sci 2014;21(05): 78-82

19 AlOtaibi F, Guiot M-C, Muanza T, Di Maio S. Giant petroclival primary intradural chordoma: case report and systematic review of the literature. J Neurol Surg Rep 2014;75(01):e160-e169

20 Ozek E, Bolukbasi FH, Baskan O, Altinoz MA, Sav A, Elmaci I. Prepontine chordoma presenting with MRI features of an epidermoid cyst: case presentation and a mini-review. Curr Med Imaging Rev 2018;14:147-150

21 Vinke RS, Lamers EC, Kusters B, van Lindert EJ. Intradural prepontine chordoma in an 11-year-old boy. A case report. Childs Nerv Syst 2016;32(01):169-173

22 Kim MJ, Park YW, Lim SM. Imaging findings of a nonenhancing intradural paramedian chordoma mimicking an epidermoid cyst. Korean J Radiol 2018;79:175-180

23 Sathe P, Mahore A, Chagla A. Cystic chordoma of the cavernous sinus. Turk Neurosurg 2019;29(02):300-302

24 Murphey MD, Walker EA, Wilson AJ, Kransdorf MJ, Temple HT, Gannon FH. From the archives of the AFIP: imaging of primary chondrosarcoma: radiologic-pathologic correlation. Radiographics 2003;23(05):1245-1278 